\title{
Validação de instrumento de participação da família no cuidado do recém-nascido hospitalizado
}

Validation of an instrument for family participation in the care of hospitalized newborns Validación de instrumento de participación de la familia en el cuidado del recién nacido hospitalizado

Carolina Queiroz de Souza Mendes',2 it https://orcid.org//0000-0002-8408-6408 Thaís Morengue Di Lello Boyamian² io https://orid.org/0000-0001-5056-0522. Nathália Norma Oliveira de Castro² io https://orcid.org/0000-0003-2876-5593. Cecília de Souza Lima Michelone² ${ }^{2}$ io https://orcid.org/0000-0002-2756-2025

Myriam Aparecida Mandetta ${ }^{2}$ in https://orcid.org/0000-0003-4399-2479 Maria Magda Ferreira Gomes Balieiro² io https://orcid.org/0000-0002-4367-5855

Mendes CQ, Boyamian TM, Como citar: CS, Mandetta MA, Balieiro MM. Validação de instrumento de participação da família no cuidado do recém-nascido hospitalizado. Acta Paul Enferm. 2020; eAPE20180282.

DOI http://dx.doi.org/10.37689/actaape/2020A0022855

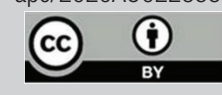

Descritores

Recém-nascido; Família; Enfermagem neonatal; Psicometria; Estudos de validação

Keywords Infant, newborn; Family; Neonatal nursing; Psychometrics; Validation studies

Descriptores

Recién nacido; Familia; Enfermería neonatal; Psicometría; Estudios de validación

\section{Submetido \\ 11 de Setembro de 2018 \\ Aceito \\ 28 de Janeiro de 2020}

Autor correspondente

Carolina Queiroz de Souza Mendes E-mail: carolqsmendes@yahoo.com.br

\section{Resumo}

Objetivo: Construir e validar um instrumento de participação da família nos cuidados do recém-nascido no contexto neonatal.

Métodos: Pesquisa metodológica. Os procedimentos de construção de instrumentos de avaliação propostos por Pasquali foram adotados para a condução das etapas teórica, empírica e analítica. Participaram do estudo piloto 40 representantes de famílias de recém-nascido hospitalizados, sendo 20 para a fase de validação semântica e 20 para o teste e resteste. Na análise dos dados o instrumento foi validado por meio da análise psicométrica e dos procedimentos estatísticos para verificar a confiabilidade, validade, estimação dos parâmetros dos itens e da medida da participação da família.

Resultados: Na fase teórica identificou-se a partir de revisão integrativa da literatura os contructos acolhimento, informação, autonomia compartilhada, autoconfiança e relacionamento colaborativo, que compõem o fenomeno participação da família no cuidado do recém-nascido hospitalizado. Dessa maneira construiu-se a versão I do instrumento de medida, contendo 32 itens, com respostas do tipo Likert, que foi submetida a avaliação entre os juízes e após duas rodadas gerou a versão II com percentual de concordância $81 \%$ e índice de validação de conteúdo (IVC) de 0,81. Na validação semântica, representantes de vinte famílias demonstraram compreensão dos itens, considerando-os relevantes, com clareza e sem dificuldade para responde-los. Na fase empírica, 0 instrumento foi aplicado com vinte representantes de famílias em que se obteve Alpha de Cronbach de 0,92. Na fase analítica, os 32 itens avaliados geraram sete fatores, cujas cargas fatoriais permitiram sua manutenção no instrumento final.

Conclusão: 0 instrumento demonstrou uma excelente consistência interna, estabilidade ao longo do tempo e itens que demonstraram pertencerem ao fenômeno analisado. Para que este indicador seja utilizado para direcionar as intervenções com família no contexto da unidade neonatal torna-se necessário ampliar a amostra da população-alvo.

\section{Abstract}

Objective: To build and validate an instrument for family participation in newborn care at the neonatal unit.

Methods: A methodological research. The construction procedures of assessment instruments proposed by Pasquali were adopted to conduct the theoretical, empirical and analytical steps. Forty representatives of hospitalized newborn families participated in the pilot study; 20 for the semantic validation phase; and 20 for the test and rest. In data analysis, the instrument was validated through psychometric analysis and statistical procedures to verify reliability, validity, estimation of items parameters, and family participation measure.

Results: In the theoretical phase, the reception, information, shared autonomy, self-confidence and collaborative 
relationships constructs, which make up the phenomenal family participation in the care of hospitalized newborns, were identified from integrative literature review. Therefore, the measurement instrument's first version was built, containing 32 items, with Likert-type responses, which was submitted to assessment by the judges. After two rounds, the second version was generated with an $81 \%$ agreement percentage and 0.81 content validation index (CVI). In the semantic validation, representatives of twenty families demonstrated an understanding of the items. They considered them relevant, clear, and had no difficulty in answering them. In the empirical phase, the instrument was applied with twenty representatives of families in which a 0.92 Cronbach's Alpha was obtained. In the analytical phase, the 32 items assessed generated seven factors, whose factor loads allowed their maintenance in the final instrument.

Conclusion: The instrument proved to have excellent internal consistency, stability over time and items that demonstrated to belong to the analyzed phenomenon. It is necessary to broaden the target population's sample for this indicator to be used to target interventions with the family at the neonatal unit.

\section{Resumen}

Objetivo: Elaborar y validar un instrumento de participación de la familia en los cuidados del recién nacido en el contexto neonatal.

Métodos: Investigación metodológica. Se adoptaron los procedimientos de elaboración de instrumentos de evaluación propuestos por Pasquali para conducir la etapa teórica, empírica y analítica. Cuarenta representantes de familias del recién nacido hospitalizado participaron en el estudio piloto, de los cuales 20 formaron parte de la fase de validación semántica y 20 del test-retest. En el análisis de los datos, el instrumento se validó por medio del análisis psicométrico y de los procedimientos estadísticos para verificar la confiabilidad, validez, estimación de los parámetros de los ítems y de la medida de participación de la familia.

Resultados: En la fase teórica, a partir de la revisión integradora de la literatura, se identificaron los constructos acogida, información, autonomía compartida, autoconfianza y relación colaborativa, que componen el fenómeno participación de la familia en el cuidado del recién nacido hospitalizado. De esta manera, se elaboró la versión I del instrumento de medida, que contenía 32 ítems con respuestas tipo Likert, y que fue evaluada por los jueces y, luego de dos rondas, se creó la versión II con un porcentaje de concordancia de 81 \% y un índice de validación de contenido (IVC) de 0,81. En la validación semántica, representantes de 20 familias demostraron comprensión de los ítems, fueron considerados relevantes, con claridad y sin dificultad para responderlos. En la fase empírica, se aplicó el instrumento a 20 representantes de familias y se obtuvo un alfa de Cronbach de 0,92. En la fase analítica, los 32 ítems evaluados generaron 7 factores, cuyas cargas factoriales permitieron que se mantengan en el instrumento final.

Conclusión: El instrumento demostró una excelente consistencia interna, estabilidad a lo largo del tiempo e ítems que pertenecen al fenómeno analizado. Para que este indicador sea utilizado para orientar las intervenciones con la familia en el contexto de la unidad neonatal, es necesario ampliar la muestra de la población destinataria.

\section{Introdução}

Com o aumento das taxas de sobrevivência, o foco do cuidado neonatal durante as últimas duas décadas passou para um paradigma centrado na melhoria da qualidade do atendimento, redução da morbidade por meio de intervençôes que incluem o cuidado desenvolvimental e o cuidado centrado na família. Esta mudança visa a redução do estresse, o apoio, a autoregulação, a promoção de experiências positivas para o recém-nascido ( $\mathrm{RN})$, bem como torna a família essencial nos cuidados de rotina. ${ }^{(1,2)}$

No Brasil, o Ministério da Saúde, por meio da Política Nacional de Humanização, estabelece o acolhimento como uma diretriz operacional do processo de trabalho em saúde que requer uma nova atitude, baseado nos pressupostos: protagonismo dos sujeitos envolvidos no processo de produção de saúde; valorização e a abertura para o encontro entre o profissional de saúde e o usuário. Esta diretriz necessita ocorrer em todos os níveis de assistência em saúde. ${ }^{(3,4)}$

O Modelo do Cuidado Centrado no Paciente e Família $^{(5,6)}$ reconhece a família como constante na vida de seus membros e a primeira responsável pelos cuidados em saúde. Sendo assim, na hospitalização deve haver parceria entre os profissionais de saúde e a família, com sua inclusão nos cuidados e nas tomadas de decisão. A família participa nos processos de planejamento, prestação e avaliação dos cuidados em conjunto com a equipe, com benefícios mútuos entre os pacientes, as famílias e os provedores de cuidado.

A hospitalização de um RN na unidade neonatal acarreta sofrimento intenso e demandas para a família, ${ }^{(7,8)}$ destacando-se as necessidades de ser informada sobre as condiçóes clínicas e de poder participar dos cuidados com o filho e das tomadas de decisão. ${ }^{(6)}$ A inclusão e a promoção da participação da família no cuidado ao RN na unidade neonatal estimula a vinculação afetiva, favorece o desenvolvimento físico e emocional do recém-nascido, ${ }^{(9,10)}$ reduz o estresse parental ${ }^{(11)}$ e promove suporte para a construção da parentalidade. ${ }^{(12)}$

Com a inserção da família no hospital, o objeto de cuidado da enfermagem é ampliado para o binômio criança-família, desencadeando a necessidade de capacitar a equipe de saúde para atender as demandas decorrentes desta prática assistencial. Os profissionais precisam entender que o cuidado à criança não deve ser desvinculado da família e das suas necessidades. ${ }^{(13,14)}$ 
$\mathrm{Na}$ ciência da enfermagem tem aumentado o número de pesquisas sobre medidas de fenômenos subjetivos da prática clínica. Dentre os instrumentos propostos observa-se uma lacuna em indicadores empíricos sobre o construto participaçáo na perspectiva do Modelo do Cuidado Centrado no Paciente e na Família (CCPF) tanto no Brasil como em outros contextos. ${ }^{(15-18)}$ Acredita-se que um instrumento brasileiro confiável de medida da participação da família poderá permitir a avaliação das intervençôes da equipe de saúde no cuidado à família no contexto neonatal.

Assim, o objetivo do estudo foi construir e validar um instrumento de Participação da Família do Recém-Nascido em Unidade Neonatal (PFRN-UN).

\section{Métodos}

Pesquisa metodológica de construção de instrumentos, que adotou os passos propostos por Pasquali quanto a condução das etapas teóricas, empíricas e analíticas. ${ }^{(19)}$

$\mathrm{Na}$ etapa teórica, uma revisão integrativa foi conduzida buscando-se identificar os indicadores de participação da família, tendo como referencial teórico o Modelo do Cuidado Centrado no Paciente e Família e referencial analítico guiado pela Análise Qualitativa de Conteúdo. Assim, os temas que emergiram da revisão integrativa compuseram os domínios e os itens do instrumento, respeitando os 12 critérios recomendados por Pasquali ${ }^{(20)}$ quais sejam: amplitude, equilíbrio, comportamento, simplicidade, clareza, relevância, precisão, modalidade, tipicidade, objetividade, variedade e credibilidade.

A partir da revisão integrativa foi construída a primeira versão do instrumento denominado Participação da Família do Recém-Nascido em Unidade Neonatal (PFRN-UN versão I). O instrumento avalia a participação da família no cuidado do $\mathrm{RN}$ na unidade neonatal e é composto por 32 itens referentes aos cinco constructos ou domínios: acolhimento, informação compartilhada, autonomia, autoconfiança e relacionamento colaborativo. As respostas do tipo Likert, variam entre: Nunca (0), Muito raramente (1), Raramente(2), Quase sempre (3) e Sempre (4). O escore de cada domínio é a somatória das respostas aos itens dividido pelo número de seus itens. $\mathrm{O}$ escore geral é a somatória das respostas de todos os itens dividido pelo numero toal de itens do instrumento (32). A interpretação do escore geral se dá pelo valor da gradação da escala likert de zero a quatro, que varia de nunca participo dos cuidados do meu filho (zero) a smpre participo dos cuidados do meu filho (quatro).

O domínio acolhimento contém sete itens sobre a abordagem e recepção da família na unidade, considerando o ambiente de cuidado, o apoio, as regras e rotinas que favorecem a presença da família e o sentimento de pertença da família (sete subitens).

A informação compartilhada, contempla seis itens relacionados a necessidade da família em ser orientada sobre a evolução das condições clínicas do $\mathrm{RN}$, de forma clara, completa, gradativa e no momento oportuno.

A autonomia é avaliada em seis itens que abordam a garantia da participação da família nas tomadas de decisão, os espaços disponibilizados para negociação com a equipe sobre os limites de atuação da família.

A autoconfiança inclui aspectos sobre a segurança e competência da família no cuidado do filho e inclui seis itens.

O relacionamento colaborativo descreve a necessidade da família em estabelecer um relacionamento com a equipe multiprofissional, em que ela se sinta acolhida, respeitada e parceira no cuidado do filho (sete itens).

A primeira versão do instrumento PFRN-UN foi submetida a um Comitê de Juízes especialistas no tema, composto por profissionais de saúde que atuavam no ensino ou em unidade de neonatologia por mais de três anos e que atenderam aos critérios de classificação de especialista segundo o Modelo de Validação de Fehring ${ }^{(21)}$ adaptado para este estudo, com pontuação igual ou superior a cinco. $\mathrm{O}$ Comitê foi composto por duas enfermeiras, um médico, uma fisioterapeuta e uma psicóloga. Além de um representante de família com filho internado na unidade neonatal, pois alguns autores ${ }^{(22)}$ consideram que a inclusão de pessoas leigas da população do estudo assegura a correção de frases e termos que não estão muito claros. 
Esse Comitê de Juízes avaliou o instrumento quanto a semântica, compreensão dos itens, redação e pertinência conceitual. Adotou-se a técnica Delphi para a busca de consenso. Um questionário foi preenchido pelos membros, com espaços para resposta de cada item em relação a sua relevância e para a emissão de sugestóes. O percentual de concordância, estabelecido entre os juízes, foi acima de $80 \%$ e o índice de validade de conteúdo acima de 0,80 . Dessa maneira, a partir do consenso gerou-se a versão II do instrumento PFRN-UN, que foi submetida à validação semântica por 20 representantes de famílias de RN internados em unidade neonatal para a análise e verificação da compreensão e clareza dos itens e dificuldade na sua utilização. Nesta etapa o critério de inclusão foi ser representante de família de recém-nascido internado na unidade neonatal há mais de 72 horas; e o de exclusão, representantes de família com distúrbios emocionais e cognitivos autorelatados, que acarretassem em barreiras na comunicação. Utilizou um questionário contendo os itens do instrumentos e espaço para a emissão de sugestóes.

A etapa empírica compreendeu a aplicação da versáo III do PFRN-UN com outros 20 representantes de famílias de recém-nascidos internados na unidade neonatal, para o teste-reteste, adotando os mesmos critérios de inclusão e exclusão.

O campo de estudo da etapa empírica da validação do instrumento de medida foi uma unidade neonatal, de um hospital de ensino da zona sul, do município de São Paulo. Esta unidade dispóe de oito leitos de terapia intensiva e 14 leitos para cuidados semi-intensivos. A equipe de saúde multiprofissional é composta por médicos neonatologistas, docentes de enfermagem e medicina, enfermeiras, técnicas de enfermagem, fisioterapeutas, psicólogo, nutricionista e alunos de pós-graduação de medicina, enfermagem, fisioterapia e fonoaudiologia.

$\mathrm{Na}$ unidade neonatal há incentivo aos pais para a realização de atividades diárias ligadas à higiene corporal do RN, como banho, troca de fraldas, curativo em coto umbilical, limpeza oral e ocular. Adota-se a estratégia do Método Canguru, o Grupo de Pais e atendimento de escuta com psicológico. Desde 2014, o modelo do cuidado centrado na fa- mília tem sido implantado com sensibilização dos profissionais e adoção de boas práticas com famílias a ser seguida nesse espaço.

Para a fase empírica utilizou-se os mesmos critérios de inclusão e exclusão das fases anteriores. As variáveis definidas para a caracterização sociodemográfica do representante da familia foram: sexo, cor, nível de escolaridade, profissão, número de filhos e composição familiar, renda familiar (em salário mínimo) e religião adotada. Já as variáveis perinatais e neonatais foram: número de consultas no pré-natal, tempo de internação no momento da entrevista com o representante da família, idade gestacional ao nascimento, peso de nascimento, sexo, nota de Apgar, tipo de parto e diagnósticos médicos.

A etapa analítica objetivou validar o instrumento por meio da análise psicométrica e dos procedimentos estatísticos para verificar a confiabilidade, validade, estimação dos parâmetros dos itens e da medida da participação da família.

Nesta etapa, as variáveis sociodemográficas dos participantes da família e o resultado da aplicação da escala foram analisados pela estatística descritiva em que, as variáveis categóricas apresentadas em frequência absoluta (n) e frequência relativa (\%); já as variáveis numéricas, por medidas apropriadas, tais como média, mediana, desvio-padrão e intervalo de confiança.

A consistência interna do conjunto de itens foi analisada pelo Alpha de Cronbach ( $\alpha$ ). O coeficiente de correlação intraclasse foi empregado para mensurar a confiabilidade de uma variável, e assim a estabilidade e a reprodutibilidade do instrumento no teste e reteste, que foi aplicado com intervalo de $72 \mathrm{~h}$. O valor varia de -1 a 1 e para melhor entendimento transformado em porcentagem. ${ }^{(19,23)}$

A medida de Kaiser-Meyer-Olkin (KMO) foi utilizada para verificar a adequação amostral para a análise fatorial.

Para a validade de constructo foram adotadas a análise fatorial exploratória e análise dos componentes principais com rotação ortogonal, pelo método Varimax, aplicado com o objetivo de maximizar a soma das variâncias das cargas da matriz fatorial. ${ }^{(19)}$ Considerou-se carga fatorial aceitável, os valores acima de 0,4 para manutenção do item no instrumento final. ${ }^{(19)}$ 
Na comparação, no teste-reteste, das respostas por questão e no escore geral do instrumento foi aplicado o teste náo-paramétrico de Wilcoxon.

O nível de significância adotado para os testes foi de 5\% ( $\mathrm{p}=0,005)$ e o pacote estatístico utilizado foi o SPSS for Windows, versão 17.0 (SPSS Inc. Chicago, Illinois), Minitab 16 e Excel Office 2010.

$\mathrm{O}$ projeto atendeu aos princípios nacionais e internacionais (Resolução CNS no 466/12) adotados com pesquisas com seres humanos; aprovado no Comitê de Ética e Pesquisa da instituição da qual os pesquisadores fazem parte, sob parecer no 449.048.

\section{Resultados}

$\mathrm{Na}$ fase teórica, o instrumento PFRN-UN apresentou em 28 itens, uma porcentagem de concordância entre os juízes de $100 \%$ e índice de validação de conteúdo (IVC) de 1,0; e em quatro itens, concordância de $83,3 \%$ e 0,83 de IVC, após duas rodadas de avaliação. $\mathrm{O}$ instrumento como um todo obteve IVC de 0,81 . Os itens com menor concordância encontravam-se nos domínios: Acolhimento (um item); Informação Compartilhada (dois itens); e Autonomia (um item). As sugestóes foram relativas ao formato redacional do texto, não havendo modificações no conteúdo conceitual de cada um dos itens do instrumento. Dessa maneira gerou-se a versão II do instrumento de PFRN-UN.

$\mathrm{Na}$ validação semântica as famílias demonstraram compreensão dos itens, considerando-os relevantes, com clareza e sem dificuldade para responder. Apenas no item 31 houve a sugestão de modificação da palavra "abertura" por "espaço", que foi acatada pelos pesquisadores. Assim, gerou-se a versão III do PFRNUN, sem mudança no seu conteúdo conceitual.

$\mathrm{Na}$ fase empírica de construção do instrumento que contou com a participação de 20 representantes da família, a totalidade era do sexo feminino $(100 \%)$, com ensino médio completo (60\%), de cor branca $(60 \%)$ e com companheiro $(75 \%)$, sendo $50 \%$ por união estável e $25 \%$ declararam-se casados. A média de idade foi de 26,9 $( \pm 6,6)$ anos. A maioria dos respondentes não tinha vínculo empregatício $(75 \%)$, adotava a religião católica $(60 \%)$ e era procedente do município de São Paulo (75\%).
A composição familiar foi em média de 4,8 $( \pm 1,2)$ pessoas por família, $2,5( \pm 1,2)$ filhos e renda média de $4,9( \pm 1,7)$ salários mínimos.

Quanto às variáveis perinatais e neonatais, as mães realizaram uma média de $9,0( \pm 4,0)$ consultas no pré-natal. A maioria dos $\mathrm{RN}$ nasceu de parto cesáreo (80\%), a média de peso ao nascer foi de 2264 $( \pm 865)$ gramas; Apgar no primeiro minuto de 5,4 $( \pm 1,3$ pontos) e 7,4 ( $\pm 1,3$ pontos) no quinto minuto; e $36^{4 / 7}\left( \pm 1^{4 / 7}\right.$ de intervalo de confiança) semanas de idade gestacional.

Os diagnósticos mais frequentes apresentados pelos RN foram prematuridade (75\%); seguidos de anomalia congênita (55\%), asfixia perinatal (15\%) e infecção congênita (10\%).

Quanto ao instrumento PFRN-UN versão III, verificou-se em três questóes diferença estatisticamente significante do teste para o reteste, nos itens: 7. "É garantida a presença de outros familiares ao meu lado na unidade" ( $\mathrm{p}=0,034) ; 25$. "Consigo entender as situaçóes vivenciadas pelo meu filho e as intervençôes que apoiam o seu desenvolvimento infantil" ( $\mathrm{p}=0,034)$; e 30. "A equipe de saúde respeita as minhas forças e compreende como vivencio e enfrento a internação do meu filho" $(\mathrm{p}=0,034)$. Isto demonstra que na maioria dos itens os resultados foram iguais, ou seja, confiáveis.

$\mathrm{Na}$ etapa analítica, a confiabilidade dos dados da aplicação do PFRN-UN versão III apresentou correlaçôes estatisticamente significantes na maioria dos itens (Tabela 1), portanto, as respostas aos itens do PFRNUN versão III são estáveis ao longo do tempo. No entanto, o item 12. "A equipe de saúde tem sido suportepara eu entender as informaçóes sobre meu filho" $(\mathrm{p}=0,207)$ não demonstrou confiabilidade no teste-reteste.

Para analisar o agrupamento dos itens do instrumento em domínios ou fatores foi inicialmente calculado o KMO, cujo resultado de 0,835 permitiu a realização da análise fatorial. O resultado do teste de Bartlett foi significante $(\mathrm{p}<0,001)$, demonstrando que os dados têm correlação entre si.

A análise fatorial pelo método dos componentes principais demonstrou a variabilidade explicada por cada fator, com valores próprios maiores que um. Os 32 itens geraram sete fatores, em que a variabilidade total foi de $89,95 \%$, o que é considerado ótimo. O primeiro fator detém $36,22 \%$ da variabilidade dos 
Tabela 1. Índice de correlação intraclasse (ICC) do teste/reteste por item do instrumento de Participação da Família do RecémNascido em Unidade Neonatal

\begin{tabular}{|c|c|c|c|}
\hline & Itens do PFRN-UM & ICC & $p$-value \\
\hline \multirow{8}{*}{ 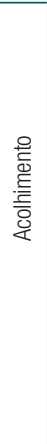 } & $\begin{array}{l}\text { q1. Tenho apoio da equipe de saúde para estar com meu filho } \\
\text { na unidade neonatal. }\end{array}$ & $92,6 \%$ & $<0,001$ \\
\hline & $\begin{array}{l}\text { q2. Conheço as características e o funcionamento do ambiente } \\
\text { da unidade neonatal. }\end{array}$ & $83,1 \%$ & $<0,001$ \\
\hline & q3. Existe um ambiente privativo para eu ficar na unidade. & $82,3 \%$ & $<0,001$ \\
\hline & q4. Tenho acomodação para estar ao lado do meu filho. & $92,3 \%$ & $<0,001$ \\
\hline & q5. 0 meu acesso à unidade é livre para estar com meu filho. & $81,4 \%$ & $<0,001$ \\
\hline & q6. Sinto-me parte da equipe de saúde da unidade. & $89,3 \%$ & $<0,001$ \\
\hline & $\begin{array}{l}\text { q7. É garantida a presença de outros familiares ao meu lado } \\
\text { na unidade. }\end{array}$ & $94,4 \%$ & $<0,001$ \\
\hline & $\begin{array}{l}\text { q8. As informações sobre meu filho são fornecidas de forma } \\
\text { clara e de fácil entendimento. }\end{array}$ & $71,4 \%$ & 0,004 \\
\hline \multirow{4}{*}{ 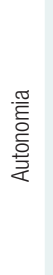 } & $\begin{array}{l}\text { q9. Recebo informações de forma completa que atendam as } \\
\text { minhas necessidades. }\end{array}$ & $72,8 \%$ & 0,003 \\
\hline & q10. Recebo as informações sobre meu filho por escrito. & $79,1 \%$ & 0,001 \\
\hline & $\begin{array}{l}\text { q11. Sou informada sobre os cuidados recebidos pelo meu } \\
\text { filho nos diversos momentos da internação (admissão, período } \\
\text { de hospitalização e alta). }\end{array}$ & $77,0 \%$ & 0,001 \\
\hline & $\begin{array}{l}\text { q12. A equipe de saúde tem sido suporte para eu entender as } \\
\text { informações sobre meu filho }\end{array}$ & $31,7 \%$ & 0,207 \\
\hline \multirow{8}{*}{ 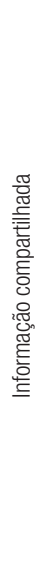 } & $\begin{array}{l}\text { q13. Tenho as informações no momento certo para tomar } \\
\text { decisão sobre o meu filho. }\end{array}$ & $61,3 \%$ & 0,023 \\
\hline & $\begin{array}{l}\text { q14. As tomadas de decisões a respeito do meu filho são } \\
\text { realizadas com a minha participação. }\end{array}$ & $86,8 \%$ & $<0,001$ \\
\hline & $\begin{array}{l}\text { q15. Posso negociar sobre o cuidado do meu filho com a } \\
\text { equipe. }\end{array}$ & $83,6 \%$ & $<0,001$ \\
\hline & $\begin{array}{l}\text { q16. Tenho abertura para discutir com a equipe de saúde } \\
\text { sobre os limites da minha participação no cuidado do meu } \\
\text { filho. }\end{array}$ & $90,6 \%$ & $<0,001$ \\
\hline & $\begin{array}{l}\text { q17. Eu sou considerada pela equipe de saúde como principal } \\
\text { responsável pelo meu filho. }\end{array}$ & $82,1 \%$ & $<0,001$ \\
\hline & $\begin{array}{l}\text { q18. Tenho minha independência garantida no cuidado do } \\
\text { meu filho. }\end{array}$ & $73,3 \%$ & 0,003 \\
\hline & $\begin{array}{l}\text { q19. As minhas idéias, crenças, valores e conhecimentos são } \\
\text { considerados pela equipe. }\end{array}$ & $86,1 \%$ & $<0,001$ \\
\hline & $\begin{array}{l}\text { q20. Sinto-me mais confiante para a alta hospitalar quando } \\
\text { cuido do meu filho. }\end{array}$ & $84,2 \%$ & $<0,001$ \\
\hline \multirow{4}{*}{ 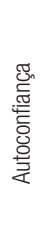 } & $\begin{array}{l}\text { q21. Sou encorajada e apoiada pela equipe de saúde para } \\
\text { cuidar do meu filho. }\end{array}$ & $82,0 \%$ & $<0,001$ \\
\hline & $\begin{array}{l}\text { q22. Percebo a equipe de saúde estimulando o meu vinculo } \\
\text { com o bebê. }\end{array}$ & $66,0 \%$ & 0,012 \\
\hline & q23. Sinto-me parceira no cuidado do meu filho. & $59,5 \%$ & 0,028 \\
\hline & $\begin{array}{l}\text { q24. Eu sou ensinada pela equipe de saúde a reconhecer as } \\
\text { individualidades do meu filho. }\end{array}$ & $79,1 \%$ & 0,001 \\
\hline \multirow{9}{*}{ 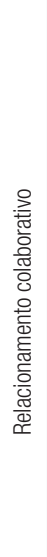 } & $\begin{array}{l}\text { q25.Consigo entender as situações vivenciadas pelo meu filho } \\
\text { e as intervenções que apoiam o seu desenvolvimento infantil. }\end{array}$ & $73,0 \%$ & 0,003 \\
\hline & q26. Eu sou acolhida pela equipe de saúde sem julgamentos. & $82,6 \%$ & $<0,001$ \\
\hline & $\begin{array}{l}\text { q27.Eu sou convidada para participar das reuniões e visitas } \\
\text { clínicas. }\end{array}$ & $78,4 \%$ & 0,001 \\
\hline & $\begin{array}{l}\text { q28. Eu considero que tenho vínculo e relacionamento de } \\
\text { parceria com a equipe de saúde. }\end{array}$ & $83,7 \%$ & $<0,001$ \\
\hline & $\begin{array}{l}\text { Q29. Eu sou compreendida pela equipe de saúde em minha } \\
\text { história social. }\end{array}$ & $82,2 \%$ & $<0,001$ \\
\hline & $\begin{array}{l}\text { q30. A equipe de saúde respeita as minhas forças e } \\
\text { compreende como vivencio e enfrento a internação do meu } \\
\text { filho. }\end{array}$ & $87,3 \%$ & $<0,001$ \\
\hline & $\begin{array}{l}\text { q31. Eu tenho espaço para ter meus questionamentos } \\
\text { respondidos. }\end{array}$ & $74,5 \%$ & 0,002 \\
\hline & q32. As minhas necessidades emocionais são atendidas. & $82,8 \%$ & $<0,001$ \\
\hline & Escore Geral & $93,1 \%$ & $<0,001$ \\
\hline
\end{tabular}

* Teste de Wilcoxon dados. As cargas fatoriais encontradas por questóes ou itens permitem que os mesmos sejam mantidos no instrumento (Tabela 2).

Tabela 2. Carga fatorial dos itens do instrumento de Participação da Família do Recém-Nascido em Unidade Neonatal em cada fator

\begin{tabular}{|c|c|c|c|c|c|c|c|}
\hline Questões & Fator 1 & Fator 2 & Fator 3 & Fator 4 & Fator 5 & Fator 6 & Fator 7 \\
\hline q.22 & 0,937 & & & & & & \\
\hline q.12 & 0,855 & & & & & & \\
\hline q.21 & 0,758 & & & & & & \\
\hline q.3 & 0,755 & & & & & & \\
\hline q30 & 0,740 & & & & & & \\
\hline q24 & 0,739 & & & & & & \\
\hline q25 & 0,703 & & & & & & \\
\hline q28 & 0,654 & & & & & & \\
\hline q11 & 0,642 & & & & & & \\
\hline q8 & 0,551 & & & & & & \\
\hline q7 & & 0,909 & & & & & \\
\hline q6 & & 0,893 & & & & & \\
\hline q5 & & 0,767 & & & & & \\
\hline q16 & & 0,698 & & & & & \\
\hline$q 4$ & & 0,696 & & & & & \\
\hline$q 1$ & & 0,659 & & & & & \\
\hline q15 & & 0,583 & & & & & \\
\hline q31 & & & 0,836 & & & & \\
\hline q32 & & & 0,831 & & & & \\
\hline $\mathrm{q} 17$ & & & 0,816 & & & & \\
\hline q29 & & & 0,712 & & & & \\
\hline q25 & & & 0,589 & & & & \\
\hline$q 18$ & & & 0,536 & & & & \\
\hline q9 & & & & 0,822 & & & \\
\hline q14 & & & & 0,775 & & & \\
\hline q19 & & & & 0,486 & & & \\
\hline q20 & & & & & $-0,878$ & & \\
\hline$q 13$ & & & & & 0,665 & & \\
\hline q24 & & & & & 0,635 & & \\
\hline$q 10$ & & & & & 0,551 & & \\
\hline q2 & & & & & & 0,845 & \\
\hline q27 & & & & & & & 0,857 \\
\hline
\end{tabular}

Método de Extração: Análise dos Componentes Principais; Método de Rotação: Varimax com Normalização de Kaiser Normalization; Rotação convertida em 14 interações.

A consistência interna do instrumento PFRNUN versão III calculada pelo Alpha de Cronbach foi alta $(0,922)$.

O escore geral da participação da família no cuidado do RN na unidade neonatal, medida pela aplicação piloto do instrumento PFRN-UN versão III, com vinte representantes de famílias apresentou mediana de 1,94 , e média de $2,01( \pm 0,48)$ evidenciando a percepção de que muito raramente a família participa do cuidado do RN na unidade neonatal.

Os escores mais altos por itens foram relacionados ao conhecimento das características e funcionamento da unidade neonatal; ao reconhecimento da 
sua responsabilidade pelo $\mathrm{RN}$; ao apoio da equipe para a permanência da família na unidade; e ao sentimento de estar preparada para a alta hospitalar do $\mathrm{RN}$, cuja resposta foi quase sempre (média de 3,0).

A consistência interna do instrumento PFRNUN versão III calculada pelo Alpha de Cronbach foi alta $(0,922)$. O escore geral da participação da família no cuidado do RN na unidade neonatal, medida pela aplicação piloto do instrumento PFRN-UN versão III, com vinte representantes de famílias apresentou mediana de 1,94 , e média de 2,01 $( \pm 0,48)$ evidenciando a percepção de que muito raramente a família participa do cuidado do $\mathrm{RN}$ na unidade neonatal. Os escores mais altos por itens foram relacionados ao conhecimento das características e funcionamento da unidade neonatal; ao reconhecimento da sua responsabilidade pelo RN; ao apoio da equipe para a permanência da família na unidade; e ao sentimento de estar preparada para a alta hospitalar do RN, cuja resposta foi quase sempre (média de 3,0). A resposta nunca dos representantes da família esteve presente nos itens relacionados ao recebimento de informação por escrito e inclusão da família nas reuniôes ou visitas clínicas. No entanto, a resposta muito raramente foi pontuada no item 19, quanto a ter suas ideias, crenças, valores e conhecimentos considerados pela equipe de saúde; item 28, ter vínculo e parceria com a equipe e item 29 , ser compreendida em sua história de vida.

\section{Discussão}

Os resultados obtidos na validação do instrumento de participação da família na unidade neonatal no estudo piloto indicaram propriedades psicométricas satisfatórias à sua utilização na prática clínica.

O instrumento PFRN-UN versão III, obteve um alto valor de Alpha de Cronbach, indicativo de excelente consistência interna; ${ }^{(18,19)}$ facilidade de compressão pelo participantes; adequação do conteúdo; e confiabilidade. Portanto, os itens do instrumento se referem ao constructo que está sendo medido, atendendo ao recomendado em estudos de validação. ${ }^{(23)}$

Dentre os 32 itens, dois constituíram-se, cada um, em fator isolado, com carga fatorial alta, o que requer a sua permanência na escala, mas indica a necessidade de maior refinamento. Dessa maneira, há necessidade de ampliação do tamanho amostral para que possa confirmar este resultado, considerando que estudos de validação recomendam o uso de amostra maior para reportar resultados mais confiáveis. ${ }^{(23)}$ Outra possibilidade, é a adoção de um cálculo amostral, a partir do teste piloto deste estudo, considerando um erro estatístico de 5\%. A ampliação do tamanho amostral poderá modificar a configuração do instrumento PFRN-UN versão III nos agrupamentos por domínios.

Os resultados da aplicaçáo deste instrumento evidenciaram dados que contribuem para a avaliação da prática relacionada ao CCPF. Neste estudo, os respondentes muito raramente percebem a família participando do cuidado do RN na unidade neonatal. Na literatura evidencia-se que, a participação da família na UTIN traz benefícios ao desenvolvimento neuropsicomotor $\mathrm{da}$ criança; o contato pele-a-pele acelera a maturação cerebral e a amamentaçáo favorece o desenvolvimento cognitivo, especialmente nos prematuros. Ademais, reduz a ansiedade, depressão e estresse parental. ${ }^{(24,25)}$

Os profissionais de saúde demonstram ainda crenças e atitudes restritivas à presença da família na unidade neonatal, e muitas vezes não percebem que, ao limitar sua participação, prejudicam o vínculo afetivo pais/ recém-nascido. No cotidiano da unidade neonatal ainda se identifica a solicitação de saída do acompanhante familiar para a execução de procedimentos invasivos, nos horários de visita médica, em razão de espaço físico reduzido e escassez de recursos humanos. ${ }^{(26,27)}$

O uso de um instrumento de medida da participação da família na unidade neonatal poderá ser um indicador da inserção da família na unidade neonatal e contribuir para a melhoria da qualidade da assistência nesse contexto.

Dessa forma, é necessário a ampliação da amostra da população-alvo, refinamento dos domínios para garantir sua confiabilidade e uso em outros contextos hospitalares.

\section{Conclusão}

O instrumento de participação da família no cuidado do recém-nascido obteve um alto índice de 
concordância entre os juízes e a população-alvo; bem como uma boa consistência interna. Os 32 itens que compóem o instrumento geraram sete fatores ou domínios, cujas cargas fatoriais permitiram sua manutenção na versão final do instrumento. Os dados advindos da aplicação do PFRN-UN podem representar um indicador na avaliação da satisfação do usuário com a organização do sistema de saúde e em relação à atuação dos profissionais na abordagem da família.

\section{Colaborações}

Mendes CQS, Boyamian TMDL, Castro NNO, Michelone CSL, Mandetta MA, Balieiro MMFG colaboraram nas etapas de concepção do estudo, análise, interpretação dos dados, redação do artigo, revisão crítica relevante do conteúdo intelectual e aprovação final da versão a ser publicada.

\section{Referências}

1. Morisaki N, Zhang X, Ganchimeg T, Vogel JP, Souza JP, Cecatti JG, et al. Provider-initiated delivery, late preterm birth and perinatal mortality: a secondary analysis of the WHO multicountry survey on maternal and newborn health. BMJ Glob Health. 2017;2(2):e000204.

2. Samra HA, McGrath JM, Wehbe M, Clapper J. Epigenetics and familycentered developmental care for the preterm infant. Adv Neonatal Care. 2012;12 Suppl 5:S2-9.

3. Martins CP, Luzio, CA. Experimentações no apoio a partir das apostas da Política Nacional de Humanização - HumanizaSUS. Interface Comunicação, Saúde, Educação. 2014; 18(1):1099-106.

4. Freitas FD, Silva RN, Araújo FP, Ferreira MA. Environment and humanization: resumption of nightingale's discourse in the national humanization. Esc Anna Nery. 2013;17(4):654-60.

5. DiGioia AM, Greenhouse PK. Creating value with the patient- and family-centered care methodology and practice: what trainees need to know, why, and strategies for medical education. AMA J Ethics. 2016;18(1):33-9.

6. Ramezani T, Hadian Shirazi Z, Sabet Sarvestani R, Moattari M. Familycentered care in neonatal intensive care unit: a concept analysis. Int J Community Based Nurs Midwifery. 2014;2(4):268-78.

7. Wang L, He JL, Fei SL. Perceived Needs of Parents of Premature Infants in NICU. West J Nurs Res. 2018;40(5):688-700.

8. Conz CA, Merighi MA, de Jesus MC. Promoção de vínculo afetivo na unidade de terapia intensiva neonatal: um desafio para as enfermeiras. Rev Esc Enferm USP. 2009;43(4):849-55.
9. O'Brien K, Bracht M, Macdonell K, McBride T, Robson K, O'Leary L, et al. A pilot cohort analytic study of Family Integrated Care in a Canadian neonatal intensive care unit. BMC Pregnancy Childbirth. 2013;13 Suppl 1:S12.

10. White-Traut RC, Rankin KM, Yoder JC, Liu L, Vasa R, Geraldo V, et al. Influence of H-HOPE intervention for premature infants on growth, feeding progression and length of stay during initial hospitalization. J Perinatol. 2015;35(8):636-41.

11. Milgrom J, Newnham C, Martin PR, Anderson PJ, Doyle LW, Hunt RW, et al. Early communication in preterm infants following intervention in the NICU. Early Hum Dev. 2013;89(9):755-62.

12. Hall SL, Hynan MT, Phillips R, Lassen S, Craig JW, Goyer E, et al. The neonatal intensive parenting unit: an introduction. J Perinatol. 2017;37(12):1259-64.

13. Melo EM, Ferreira PL, Lima RA, Mello DF. The involvement of parents in the healthcare provided to hospitalzed children. Rev Lat Am Enfermagem. 2014;22(3):432-9.

14. Galarza-Winton ME, Dicky T, O'Leary L, Lee SK, O'Brien K. Implementing family-integrated care in the NICU: educating nurses. Adv Neonatal Care. 2013;13(5):335-40.

15. Cruz AC, Angelo M, Santos BP. Self-efficacy scale for the establishment of good relationships with families in neonatal and pediatric hospital settings. Rev Esc Enferm USP. 2017;51:e03222.

16. Quemelo PR, Milani D, Bento VF, Vieira ER, Zaia JE. Literacia em saúde: tradução e validação de instrumento para pesquisa em promoção da saúde no Brasil. Cad Saude Publica. 2017;33(2):e00179715.

17. Arigliani M, Castriotta L, Pusiol A, Titolo A, Petoello E, Brun Peressut A, et al. Measuring empathy in pediatrics: validation of the Visual CARE measure. BMC Pediatr. 2018;18(1):57.

18. Andrade RC,LeiteAC,AlvarengaWA,Martimiano RR,Santos CB, Nascimento LC. Tradução e adaptação cultural do Needs of Parents Questionnaire (NPQ) para uso no Brasil. Esc Anna Nery. 2018;22(1):e20170104.

19. Pasquali L. Princípios de elaboração de escalas psicológicas. Rev Psiquiatr Clin (Santiago). 1998;25(5):206-13.

20. Pasquali L. Psicometria. Rev Esc Enferm USP. 2009; 43(Esp):992-9.

21. Fehring RJ. The Fehring model. In: Carrol-Jonhnson RM, Paquete M, editores. Classification of nursing diagnoses: proceedings of the Tenth Conference; 1994. Philadelphia: J.B. Limppincott; 1994. p. 55-62.

22. Coluci MZ, Alexandre NM, Milani D. Construção de instrumentos de medida na área da saúde. Cien Saude Colet. 2015;20(3):925-36.

23. Streiner DL, Kottner J. Recommendations for reporting the results of studies of instrument and scale development and testing. J Adv Nurs. 2014;70(9):1970-9.

24. Skene C, Gerrish K, Price F, Pilling E, Bayliss P. Developing familycentred care in a neonatal intensive care unit: an action research study protocol. J Adv Nurs. 2016;72(3):658-68.

25. Tobiano G, Marshall A, Bucknall T, Chaboyer W. Patient participation in nursing care on medical wards: an integrative review. Int J Nurs Stud. 2015;52(6):1107-20.

26. Ferreira CA, Balbino FS, Balieiro MM, Mandetta MA. Family presence during cardiopulmonary resuscitation and invasive procedures in children. Rev Paul Pediatr. 2014;32(1):107-13.

27. Reis CA, Andrade AC, Manzo BF, Couto DL, Duarte ED. The familycentered care practices in newborn unit nursing perspective. Esc Anna Nery. 2015;19(4):629-34. 